\title{
Solução da equação de advecção-difusão tridimensional pelo método GIADMT para dois termos de contragradiente
}

\author{
Solution of the three-dimensional advection-diffusion equation by the method \\ GIADMT for two countergradient terms
}

\author{
Karine Rui ${ }^{1}$ e Camila Pinto da Costa ${ }^{2}$ \\ ${ }^{1}$ Mestranda, Programa de Pós-Graduação em Modelagem Matemática, Universidade \\ Federal de Pelotas, Pelotas, Brasil \\ ${ }^{2}$ Doutora, Departamento de Matemática e Estatística, Universidade Federal de Pelotas, \\ Pelotas, Brasil
}

\begin{abstract}
Resumo
Neste trabalho, apresentamos a resolução da equação de advecção-difusão tridimensional estacionária, por meio da técnica GIADMT, considerando o fechamento não-local para o fluxo turbulento, utilizando duas parametrizações diferentes para o contragradiente, uma proposta por Cuijpers e Holtslag (1998) e a outra proposta por Roberti et al. (2004). A concentração de poluentes é estimada e comparada com os dados observados no experimento de Copenhagen utilizando diferentes parametrizações para o coeficiente de difusão turbulento vertical.
\end{abstract}

Palavras-chave: Fechamento não-local, contragradiente, GIADMT, equação de advecção-difusão.

\begin{abstract}
In this work, we present the resolution of the three-dimensional stationary advection-diffusion equation, through the GIADMT technique, considering the nonlocal closure for turbulent flow, using two different parameterization for the countergradient, one proposal by Cuijpers e Holtslag (1998) and another proposed by Roberti et al. (2004). The concentration of pollutants is estimated and compared with the observed data in Copenhagen experiment using different parameterization for the vertical turbulent diffusion coefficient.
\end{abstract}

Keywords: Nonlocal closure, countergradient, GIADMT, advection-diffusion equation. 


\section{Introdução}

A concentração de poluentes ao nível do solo pode ser estimada através da equação de advecção-difusão, que é obtida a partir da parametrização dos fluxos turbulentos na equação da continuidade de massa. Para solucionar esta equação é preciso determinar o fechamento das equações dos fluxos turbulentos. Uma das hipóteses mais utilizadas para resolver o fechamento da equação é a hipótese de transporte por gradiente, ou teoria K, que assume que a turbulência é proporcional à magnitude do gradiente de concentração média, ou seja, os fluxos turbulentos são dirigidos para baixo do gradiente médio:

$$
\begin{aligned}
& \overline{u^{\prime} c^{\prime}}=-K_{x} \frac{\partial \bar{c}}{\partial x}, \\
& \overline{v^{\prime} c^{\prime}}=-K_{y} \frac{\partial \bar{c}}{\partial y}, \\
& \overline{w^{\prime} c^{\prime}}=-K_{z} \frac{\partial \bar{c}}{\partial z},
\end{aligned}
$$

onde $\bar{c}$ denota a concentração média de poluentes, $\overline{u^{\prime} c^{\prime}}$, $\overline{v^{\prime} c^{\prime}}$ e $\overline{w^{\prime} c^{\prime}}$ representam, respectivamente, os fluxos turbulentos de contaminantes nas direções $x, y$ e $z$, e $K_{x}$, $K_{y}$ e $K_{z}$ são, respectivamente, os coeficientes de difusão horizontal, lateral e vertical.

A teoria $\mathrm{K}$ é um método para parametrizar os efeitos da turbulência mista com base em como os pequenos turbilhões serão quantidades misturadas ao longo de um gradiente local das quantidades transportadas, $\mathrm{CO}^{-}$ nhecido como fechamento local.

Porém esta teoria não leva em conta o caráter não homogêneo da turbulência da camada limite convectiva (CLC), ou seja, quando os movimentos convectivos dominam o transporte e o processo difusivo. Os fluxos de contragradiente são pensados para ser um indicativo de turbilhões de escala na CLC e são chamados de fluxos não-locais. Na parte superior da CLC o fluxo de temperatura potencial é ao contrário do gradiente de perfil de temperatura potencial do meio (Deardorff, 1972). O calor é transferido em grandes escalas por movimentos convectivos de turbilhões que se manisfestam na forma de correntes de ar ascendentes e correntes de ar descendentes. Os mesmos turbilhões também podem transportar material para cima e para baixo na CLC independentemente dos gradientes médios de concentração (Arya, 1999). O gradiente de temperatura potencial do meio e o fluxo trocam de sinal em diferentes níveis, introduzindo na CLC uma certa região onde eles tem o mesmo sinal (Deardorff, 1972).

Para considerar esse caráter no processo de dispersão de poluentes, o fechamento da turbulência não-Fickiano ou fechamento não-local é considerado. Deardorff (1966, 1972) propôs a inclusão de um termo de contragradiente para descrever a difusão também nas regiões superiores da CLC:

$$
\overline{w^{\prime} c^{\prime}}=-K_{z}\left(\frac{\partial \bar{c}}{\partial z}-\gamma\right)
$$

onde $\gamma$ é o termo de contragradiente.

Neste trabalho, resolvemos a equação de advecçãodifusão tridimensional em estado estacionário, pelo método GIADMT (Generalized Integral Advection Diffusion Multilayer Technique) (Costa et al., 2006), considerando o fechamento não-local da turbulência utilizando duas diferentes parametrizações para o termo de contragradiente propostas por Cuijpers e Holtslag (1998) e Roberti et al. (2004). Validamos a solução da equação através dos dados medidos no experimento de Copenhagen empregando diferentes coeficientes de difusão vertical.

\section{Resolução via GIADMT}

A equação de advecção-difusão que modela a dispersão de poluentes na atmosfera pode ser escrita como:

$$
\bar{u} \frac{\partial \bar{c}}{\partial x}=-\frac{\partial \overline{v^{\prime} c^{\prime}}}{\partial y}-\frac{\partial \overline{w^{\prime} c^{\prime}}}{\partial z}+S,
$$

onde $\bar{u}$ denota a velocidade média do vento na direção horizontal e $S$ é o termo fonte.

Para o termo do contragradiente usamos duas parametrizações diferentes. A parametrização proposta por Cuijpers e Holtslag em 1998 (Cuijpers e Holtslag, 1998):

$$
\gamma_{1}=\frac{b w_{*}^{2} \bar{c}}{\sigma_{w}^{2} h}
$$

onde $b$ é uma constante, $w_{*}$ é a velocidade escalar convectiva, $\sigma_{w}$ é o desvio padrão vertical da velocidade turbulenta, $h$ é a altura da CLC.

E a parametrização proposta por Roberti et al. em 2004 (Roberti et al., 2004):

$$
\gamma_{2}=0.085 \frac{q_{w}}{\Psi}\left(\frac{h}{z}\right)^{2 / 3} \frac{\bar{c}}{h},
$$

onde $\Psi=0.913$ é a função de dissipação adimensional e $q_{w}$ é a função de estabilidade dada por:

$$
q_{w}=z\left[0.594 h\left(1-e^{-4(z / h)}-0.0003 e^{8(z / h)}\right)\right]^{-1} .
$$

Para facilitar a notação, definimos:

$$
\beta_{1}=\frac{b w_{*}^{2}}{\sigma_{w}^{2} h},
$$

e, assim, temos que:

$$
\gamma_{1}=\beta_{1}(z) \bar{c}(x, y, z),
$$


de forma análoga, definimos:

$$
\beta_{2}=\frac{0.085}{h} \frac{q_{w}}{\Psi}\left(\frac{h}{z}\right)^{2 / 3}
$$

assim, podemos escrever:

$$
\gamma_{2}=\beta_{2}(z) \bar{c}(x, y, z)
$$

Substituindo as equações (1) e (2) em (3), obtemos:

$$
\bar{u} \frac{\partial \bar{c}}{\partial x}=\frac{\partial \bar{c}}{\partial y}\left(K_{y} \frac{\partial \bar{c}}{\partial y}\right)+\frac{\partial \bar{c}}{\partial z}\left(K_{z} \frac{\partial \bar{c}}{\partial z}\right)+K_{z} \beta_{\alpha} \frac{\partial \bar{c}}{\partial z}
$$

para $0<z<h, 0<y<L_{y}$ e $x>0$, onde $L_{y}$ é a distância da fonte e $\alpha=1$ ou 2. A equação (6) está sujeita as seguintes condições de contorno:

$$
\begin{aligned}
-K_{z}\left(\frac{\partial \bar{c}}{\partial z}-\gamma_{\alpha}\right)=0 \quad \text { em } z=0, h \\
-K_{y}\left(\frac{\partial \bar{c}}{\partial y}\right)=0 \quad \text { em } y=0, L_{y},
\end{aligned}
$$

e a condição de fonte, considerando uma taxa de emissão contínua de poluente, $Q$, é escrita por:

$$
\bar{u} \bar{c}(0, y, z)=Q \delta\left(z-H_{S}\right) \delta\left(y-y_{0}\right) \quad \text { em } x=0,
$$

onde $\delta$ é a função Delta de Dirac, $H_{s}$ é a altura da fonte e $y_{0}$ é a posição da fonte em $y$.

A solução do problema (6) é obtida fazendo uso do método GIADMT (Costa et al., 2006) que é uma combinação dos métodos ADMM (Advection Diffusion Multilayer Method) e GITT (Generalized Integral Transform Technique).

Primeiramente, devemos levar em conta a dependência dos coeficientes de difusão, velocidade do vento e o termo de contragradiente em relação a altura de variável $z$. Para este efeito, através do método ADMM, discretizamos a altura $h$ da CLC em $N$ subcamadas de forma que no interior de cada subcamada $\bar{u}, K_{\tau}$ e $\beta_{\alpha}$ assumem valores médios:

$$
\begin{aligned}
K_{\tau_{n}} & =\frac{1}{z_{n-1}-z_{n}} \int_{z_{n-1}}^{z_{n}} K_{\tau}(z) \mathrm{d} z \\
\bar{u}_{n} & =\frac{1}{z_{n-1}-z_{n}} \int_{z_{n-1}}^{z_{n}} \bar{u}(z) \mathrm{d} z, \\
\beta_{\alpha_{n}} & =\frac{1}{z_{n-1}-z_{n}} \int_{z_{n-1}}^{z_{n}} \beta_{\alpha}(z) \mathrm{d} z,
\end{aligned}
$$

para $n=1: N$ e $\tau=x, y, z$.

Consideramos também condições de continuidade para a concentração e de fluxo de concentração nas interfaces dadas por:

$$
\bar{c}_{n}=\bar{c}_{n+1}
$$

e

$$
K_{z_{n}}\left(\frac{\partial \bar{c}_{n}}{\partial z}-\gamma_{\alpha_{n}}\right)=K_{z_{n+1}}\left(\frac{\partial \bar{c}_{n+1}}{\partial z}-\gamma_{\alpha_{n+1}}\right),
$$

para $z=z_{n}$ e $n=1,2, \ldots(N-1)$.

Sendo assim, o problema (6) é reformulado como um conjunto de problemas advectivo-difusivo com parâmetros constantes, onde para cada subcamada genérica temos:

$$
\bar{u}_{n} \frac{\partial \bar{c}_{n}}{\partial x}=K_{y_{n}} \frac{\partial^{2} \bar{c}_{n}}{\partial y^{2}}+K_{z_{n}} \frac{\partial^{2} \bar{c}_{n}}{\partial z^{2}}+K_{z_{n}} \beta_{\alpha_{n}} \frac{\partial \bar{c}_{n}}{\partial z},
$$

com as seguintes condições de contorno:

$$
\begin{array}{r}
-K_{z_{n}}\left(\frac{\partial \bar{c}_{n}}{\partial z}-\gamma_{\alpha_{n}}\right)=0 \quad \text { em } \quad z=0, h, \\
-K_{y_{n}}\left(\frac{\partial \bar{c}_{n}}{\partial y}\right)=0 \quad \text { em } y=0, L_{y},
\end{array}
$$

e a condição de fonte:

$$
\bar{u}_{n} \bar{c}_{n}(0, y, z)=Q \delta\left(z-H_{s}\right) \delta\left(y-y_{0}\right) \quad \text { em } x=0,
$$

com $z_{n-1} \leq z \leq z_{n}$ para $n=1: N$, onde $N$ denota o número de subcamadas e $\bar{c}_{n}$ denota a concentração média na enésima subcamada.

Para resolver o problema (7), aplicamos o método GITT na direção $y$. Seguindo o formalismo da GITT começamos a expandir a variável $\bar{c}_{n}(x, y, z)$ pela série:

$$
\bar{c}_{n}(x, y, z)=\sum_{j=0}^{\infty} \frac{\overline{\bar{c}}_{j_{n}}(x, z) \psi_{j}(y)}{\sqrt{N_{j}}}=0,
$$

onde $\psi_{j}(y)=\cos \left(\lambda_{j} y\right)$ são as autofunções do problema auxiliar de Sturm-Liouville na variável $y, \lambda_{j}=j \pi / L_{y}$ os autovalores correspondentes e $N_{j}=\int_{y} \psi_{j}^{2}(y) \mathrm{d} y$ é a norma.

Substituindo a equação (8) em (7) e usando a propriedade de ortogonalidade das autofunções chega-se na seguinte equação:

$$
\begin{aligned}
-\bar{u}_{n} \frac{\partial \overline{\bar{c}}_{j_{n}}(x, z)}{\partial x}-K_{y_{n}} \lambda_{j}^{2} \overline{\bar{c}}_{j_{n}}(x, z)+ & K_{z_{n}} \frac{\partial^{2} \overline{\bar{c}}_{j_{n}}(x, z)}{\partial z^{2}}+ \\
& +K_{z_{n}} \beta_{\alpha_{n}} \frac{\partial \overline{\bar{c}}_{j_{n}}(x, z)}{\partial z}=0,
\end{aligned}
$$

Fazendo o mesmo procedimento na condição de contorno (7a) e condição de fonte (7c), obtém-se:

$$
\begin{aligned}
K_{z_{n}}\left(\frac{\partial \overline{\bar{c}}_{j_{n}}(x, z)}{\partial z}-\sqrt{L_{y}} \gamma_{\alpha_{n}}\right) & =0 \text { em } z=0, h \text { para } j=0, \\
K_{z_{n}}\left(\frac{\partial \overline{\bar{c}}_{j_{n}}(x, z)}{\partial z}\right) & =0 \text { em } z=0, h \text { para } j \neq 0,
\end{aligned}
$$

e

$$
\overline{\bar{c}}_{j_{n}}(0, z)=\frac{Q \delta\left(z-H_{S}\right)}{\bar{u}_{n}} \frac{\psi_{j}\left(y_{0}\right)}{\sqrt{N_{j}}}
$$


Aplicando na equação (9) a Transformada de Laplace na variável $x\left(\mathscr{L}\left\{\overline{\bar{c}}_{j_{n}}(x, z)\right\}=\overline{\bar{C}}_{j_{n}}(s, z)\right)$ e resolvendo a equação diferencial ordinária resultante, obtemos:

$$
\begin{aligned}
\overline{\bar{C}}_{j_{n}}(s, z)=A_{n} e^{\left(F_{n}+R_{n}\right) z}+B_{n} e^{\left(F_{n}-R_{n}\right) z}+ \\
+\frac{Q \psi_{j}\left(y_{0}\right)}{2 R_{n} K_{z_{n}} \sqrt{N_{j}}}\left[e^{\left(F_{n}-R_{n}\right)\left(z-H_{s}\right)}+\right. \\
\left.+e^{\left(F_{n}+R_{n}\right)\left(z-H_{s}\right)}\right] H\left(z-H_{s}\right),
\end{aligned}
$$

para $n=1 \ldots N-1$, onde $H$ é a função de Heaviside e o último termo da equação (10) é a solução particular válida somente na região de emissão do poluente. Com

$$
F_{n}=-\frac{\beta_{\alpha_{n}}}{2},
$$

e

$$
R_{n}=\frac{\sqrt{\beta_{\alpha_{n}}^{2}+4\left(\bar{u}_{n} s+K_{y_{n}} \lambda_{j}^{2}\right) / K_{z_{n}}}}{2} .
$$

Para se determinar as constantes $A_{n}$ e $B_{n}$, aplica-se as $(2 N-2)$ condições de continuidade de interfaces e as condições de contorno chegando a sistema linear de dimensão $2 N$ dado por $M v=b$ :

$M=$

$$
\left[\begin{array}{cccccccccc}
M_{11} & M_{12} & 0 & 0 & 0 & 0 & 0 & 0 & \ldots & 0 \\
M_{21} & M_{22} & M_{23} & M_{24} & 0 & 0 & 0 & 0 & \ldots & 0 \\
M_{31} & M_{32} & M_{33} & M_{34} & 0 & 0 & 0 & 0 & \ldots & 0 \\
0 & 0 & M_{43} & M_{44} & M_{45} & M_{46} & 0 & 0 & \ldots & 0 \\
0 & 0 & M_{53} & M_{54} & M_{55} & M_{56} & 0 & 0 & \ldots & 0 \\
0 & 0 & 0 & 0 & M_{65} & M_{66} & M_{67} & M_{68} & \ldots & 0 \\
0 & 0 & 0 & 0 & M_{75} & M_{76} & M_{77} & M_{78} & \ldots & 0 \\
\vdots & \vdots & \vdots & \vdots & \vdots & \vdots & \vdots & \vdots & \vdots & \vdots \\
0 & 0 & 0 & 0 & 0 & 0 & \omega_{1} & \omega_{2} & \omega_{3} & \omega_{4} \\
0 & 0 & 0 & 0 & 0 & 0 & 0 & 0 & \omega_{5} & \omega_{6}
\end{array}\right]
$$

onde

$$
\begin{aligned}
& \omega_{1}=M_{2 N-1,2 N-3}, \\
& \omega_{2}=M_{2 N-1,2 N-2,} \\
& \omega_{3}=M_{2 N-1,2 N-1,} \\
& \omega_{4}=M_{2 N-1,2 N,} \\
& \omega_{5}=M_{2 N, 2 N-1,} \\
& \omega_{6}=M_{2 N, 2 N} .
\end{aligned}
$$

$$
v=\left[\begin{array}{llllllll}
A_{1} & B_{1} & A_{2} & B_{2} & \cdots & \cdots & A_{N} & B_{N}
\end{array}\right]^{T},
$$

e

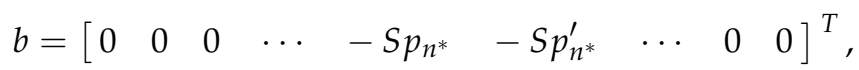

onde $n^{*}$ indica a região de emissão do poluente, $S p_{n^{*}}$ é a solução particular da equação (9) e $S p_{n^{*}}^{\prime}$ é a derivada da solução particular, ambas são aplicadas somente na região de emissão.

$$
\begin{aligned}
& S p_{n^{*}}=\frac{Q \psi_{j}\left(y_{0}\right)}{2 R_{n^{*}} K_{z_{n^{*}}} \sqrt{N_{j}}} \cdot \\
& \cdot\left[e^{\left[F_{n^{*}}-R_{n^{*}}\right]\left(z-H_{s}\right)}-e^{\left.\left[F_{n^{*}}+R_{n^{*}}\right]\left(z-H_{s}\right)\right]},\right. \\
& S p_{n^{*}}^{\prime}=\frac{Q \psi_{j}\left(y_{0}\right)}{2 R_{n^{*}} K_{z_{n^{*}}} \sqrt{N_{j}}}\left[\left(F_{n^{*}}-R_{n^{*}}\right) e^{\left[F_{n^{*}}-R_{n^{*}}\right]\left(z-H_{s}\right)}-\right. \\
&\left.-\left(F_{n^{*}}-R_{n^{*}}\right) e^{\left[F_{n^{*}+R_{n^{*}}}\right]\left(z-H_{s}\right)}\right] .
\end{aligned}
$$

A matriz $M$, é definida como segue:

Para $j=0$ :

$$
\begin{aligned}
M_{11} & =F_{1}+R_{1}-\beta_{\alpha_{1}} \sqrt{L_{y}}, \\
M_{12} & =F_{1}-R_{1}-\beta_{\alpha_{1}} \sqrt{L_{y}}, \\
\text { e para } j & \neq 0: \\
M_{11} & =F_{1}+R_{1}, \\
M_{12} & =F_{1}-R_{1} .
\end{aligned}
$$

Para $n=1,2,3, \ldots, N-1$ :

$$
\begin{aligned}
M_{2 n, 2 n-1} & =e^{\left[F_{n}+R_{n}\right] z_{n}} \\
M_{2 n, 2 n} & =e^{\left[F_{n}-R_{n}\right] z_{n}} \\
M_{2 n, 2 n+1} & =-e^{\left[F_{n+1}+R_{n+1}\right] z_{n}} \\
M_{2 n, 2 n+2} & =-e^{\left[F_{n+1}-R_{n+1}\right] z_{n}} \\
M_{2 n+1,2 n-1} & =K_{z_{n}}\left[F_{n}+R_{n}-\beta_{\alpha_{n}}\right] e^{\left[F_{n}+R_{n}\right] z_{n}} \\
M_{2 n+1,2 n} & =-K_{z_{n}}\left[F_{n}-R_{n}-\beta_{\alpha_{n}}\right] e^{\left[F_{n}-R_{n}\right] z_{n}} \\
M_{2 n+1,2 n+1} & =-K_{z_{n+1}}\left[F_{n+1}+R_{n+1}-\beta_{\left.\alpha_{n+1}\right]} \cdot\right. \\
M_{2 n+1,2 n+2} & =-K_{z_{n+1}}\left[F_{n+1}-R_{n+1}\right] z_{n} \\
& -\beta_{\left.\alpha_{n+1}\right]} \cdot e^{\left[F_{n+1}-R_{n+1}\right] z_{n}}
\end{aligned}
$$

e, por fim, para $j=0$ :

$$
\begin{aligned}
M_{2 N, 2 N-1} & =\left[F_{N}+R_{N}-\beta_{\alpha_{N}} \sqrt{L_{y}}\right] e^{\left[F_{N}+R_{N}\right] z_{N}}, \\
M_{2 N, 2 N} & =\left[F_{N}-R_{N}-\beta_{\alpha_{N}} \sqrt{L_{y}}\right] e^{\left[F_{N}-R_{N}\right] z_{N}},
\end{aligned}
$$

e para $j \neq 0$ :

$$
\begin{aligned}
M_{2 N, 2 N-1} & =\left[F_{N}+R_{N}\right] e^{\left[F_{N}+R_{N}\right] z_{N}}, \\
M_{2 N, 2 N} & =\left[F_{N}-R_{N}\right] e^{\left[F_{N}-R_{N}\right] z_{N}} .
\end{aligned}
$$

Por fim, o sistema $M v=b$ é resolvido utilizando o método de Eliminação de Gauss (Burden e Faires, 2003). 
A concentração final de poluentes é obtida invertendo numericamente a concentração transformada pela aplicação do Algoritmo de Fixed-Talbot (Abate e Valkó, 2004), obtendo a solução:

$$
\begin{gathered}
\bar{c}_{n}(x, y, z)=\sum_{j=0}^{\infty} \frac{\psi_{j}(y)}{\sqrt{N_{j}}}\left\{\frac { r } { M ^ { * } } \left[\frac{1}{2} \overline{\bar{c}}_{j_{n}}(r, z) e^{r x}+\right.\right. \\
\left.\left.+\sum_{k=1}^{M^{*}-1} \operatorname{Re}\left[e^{x s\left(\theta_{k}\right)} \overline{\bar{c}}_{j_{n}}\left(S\left(\theta_{k}\right), z\right)\left(1+i \bar{w}\left(\theta_{k}\right)\right)\right]\right]\right\},
\end{gathered}
$$

onde:

$S\left(\theta_{k}\right)=r \theta(\cot \theta+i), \bar{\omega}\left(\theta_{k}\right)=\theta_{k}+\left(\theta_{k} \cot \theta_{k}-1\right) \cot \theta_{k}$ $\theta_{k}=\frac{k \pi}{M^{*}},-\pi<\theta<+\pi, r$ é um parâmetro baseado em experimentos numéricos, $i \in \mathbb{C}$ e $M^{*}$ é a precisão do número de dígitos decimais.

Neste trabalho foram utilizadas três parametrizações da camada limite para o coeficiente de difusão vertical, uma parametrização para o coeficiente de difusão lateral e uma parametrização para o perfil da velocidade do vento.

Empregamos para o coeficiente de difusão vertical a fórmula proposta por Degrazia et al. em 1997 (Degrazia et al., 1997) da seguinte forma:

$$
\begin{aligned}
\frac{K_{z}}{w_{*} h}= & 0.22\left(\frac{z}{h}\right)^{1 / 3}\left(1-\frac{z}{h}\right)^{1 / 3} . \\
& \cdot\left[1-\exp \left(-\frac{4 z}{h}\right)-0.0003 \exp \left(\frac{8 z}{h}\right)\right],
\end{aligned}
$$

onde $w_{*}$ é a velocidade convectiva.

A fórmula proposta por Degrazia et al. em 2001 (Degrazia et al., 2001) dada por:

$$
\begin{aligned}
\frac{K_{z}}{w_{*} h}=\frac{0.09 c_{w}^{1 / 2} \psi^{1 / 3}(z / h)^{4 / 3}}{\left(f_{m}^{*}\right)_{w}^{4 / 3}} . \\
\cdot \int_{0}^{\infty} \frac{\sin \left[\frac{7.84 c_{w}^{1 / 2} \psi^{1 / 3}\left(f_{m}^{*}\right)_{w}^{2 / 3} X n}{(z / h)^{2 / 3}}\right]}{\left(1+n^{\prime}\right)^{5 / 3}} \frac{\mathrm{d} n^{\prime}}{n^{\prime}},
\end{aligned}
$$

onde $\psi=1.26 \exp (-z / 0.8 h)$ é a taxa de dissipação molecular adimensional associada a produção da pluma, $c_{v, w}=0.4, n^{\prime}=\hat{n}\left(1.5 z / u\left(f_{m}^{*}\right)_{w}\right)$ sendo $\hat{n}$ a frequência e $X$ é o tempo adimensional uma vez que é a taxa de tempo de viagem $x / u$ e a escala de tempo convectivo $h / w_{*},\left(f_{m}^{*}\right)_{w}=z /\left(\lambda_{m}\right)_{w}$ é a frequência adimensional do pico espectral, $\left(\lambda_{m}\right)_{w}=1.8 h[1-\exp (-4 z / h)-$ $0.0003 \exp (8 z / h)]$ é o comprimento de onda associado ao máximo do espectro vertical turbulento.

E a fórmula proposta por Pleim e Chang em 1992 (Pleim e Chang, 1992):

$$
K_{z}=k w_{*} z(1-z / h),
$$

onde $k=0.4$ é a constante de von Kármán.

Para o coeficiente de difusão lateral usamos a fórmula sugerida por Degazia et al. em 1997 (Degrazia et al., 1997):

$$
K_{y}=\frac{\sqrt{\pi} \sigma_{v} z}{16\left(f_{m}\right)_{v} q_{v}},
$$

com

$$
\sigma_{v}^{2}=\frac{0.98 c_{v}}{\left(f_{m}\right)_{v}^{2 / 3}}\left(\frac{\psi_{\epsilon}}{q_{v}}\right)^{2 / 3}\left(\frac{z}{h}\right)^{2 / 3} w_{*}^{2},
$$

$$
\psi_{\epsilon}^{1 / 3}=\left[\left(1-\frac{z}{L}\right)^{2}\left(-\frac{z}{L}\right)^{-2 / 3}+0.75\right]^{1 / 2}
$$

onde $u_{*}$ é a velocidade de fricção, $L$ é o comprimento de Monin-Obukhov, $\sigma_{v}$ é o desvio padrão da componente da velocidade turbulenta longitudinal, $\left(f_{m}\right)_{v}=0.16$ é o pico de onda lateral, $\psi_{\epsilon}$ é a função da taxa de dissipação molecular adimensional, $q_{v}=4.16 z / h$ é a função de estabilidade e $c_{v}=0.4$.

O desvio padrão da componente de velocidade turbulenta vertical, no termo do contragradiente $\gamma_{1}$, é dado por Sorbjan em 1989 (Sorbjan, 1989):

$$
\sigma_{w}^{2}=1.8\left(\frac{z}{h}\right)^{2 / 3}\left(1-\frac{z}{h}\right)^{2 / 3} w_{*}^{2} .
$$

Para o perfil da velocidade do vento utilizamos a parametrização proposta por Panofsky e Dutton em 1984 (Panofsky e Dutton, 1984):

$$
\frac{\overline{u_{z}}}{\overline{u_{1}}}=\left(\frac{z}{z_{1}}\right)^{p}
$$

onde $\overline{u_{z}}$ e $\overline{u_{1}}$ são as velocidades médias do vento nas alturas $z$ e $z_{1}$, enquanto $p$ é um expoente que está relacionado com a intensidade da turbulência (Irwin, 1979).

A fim de validar a solução da equação com os termos de contragradiente avaliamos a concentração ao nível do solo calculada pela equação (11) com dados observados no experimento de Copenhagen. O conjunto de dados de Copenhagen podem ser encontrados em Gryning e Lyck (2002) e foram medidos através de experimentos realizados em condições atmosféricas moderadamente instáveis na região norte de Copenhagen. Foi liberado Hexafluoreto de Enxofre $\left(S F_{6}\right)$ de uma fonte de $115 \mathrm{~m}$ de altura, com taxa de emissão de $100 \mathrm{~g} / \mathrm{s}$. Os dados foram coletados de três arcos posicionados de $2 \mathrm{a} 6 \mathrm{~km}$ do ponto no qual ocorreu a liberação do traçador. $\mathrm{O}$ comprimento de rugosidade considerado é de $0.6 \mathrm{~m}$. Uma análise estatística, descrita por Hanna em 1989 (Hanna, 1989), foi realizada nos resultados obtidos. 


\section{Resultado e discussões}

A comparação dos dados observados no experimento confrontado com os dados simulados no modelo foi realizada para diferentes parametrizações do coeficiente de difusão e os dois termos de contragradiente.

A Tabela 1 mostra o desempenho do modelo usando um procedimento de avaliação estatística descrito por Hanna (1989) da seguinte maneira:

- Erro quadrático médio normalizado:

Nmse $=\overline{\left(C_{o}-C_{p}\right)^{2}} / \overline{C_{0} C_{p}}$.

(valor ideal: Nmse $=0$ )

- Coeficiente de correlação:

Cor $=\left[\overline{\left(C_{o}-\overline{C_{o}}\right)\left(C_{p}-\overline{C_{p}}\right)}\right] / \sigma_{o} \sigma_{p}$.

(valor ideal: $\mathrm{Cor}=1$ )

- Fator de dois:

$F a 2=C_{p} / C_{o} \in[0.5,2]$.

(valor ideal: $F a 2=1$ )

- Erro fracional:

$F b=\left(\overline{C_{o}}-\overline{C_{p}}\right) /\left(0.5\left(\overline{C_{o}}+\overline{C_{p}}\right)\right)$.

(valor ideal: $F b=0$ )

- Desvio padrão fracional:

$F s=\left(\sigma_{o}-\sigma_{p}\right) /\left(0.5\left(\sigma_{o}+\sigma_{p}\right)\right)$.

(valor ideal: $F s=0$ )

onde $o$ indica as quantidades observadas nos experimentos, $p$ as quantidades preditas pelo modelo, $\mathrm{C}$ a concentração de poluentes e $\sigma$ é o desvio padrão. Observando os índices estatísticos obtidos podemos afirmar que o modelo simula satisfatoriamente as concentrações observadas com valores de Nmse, $\mathrm{Fb}$ e $\mathrm{Fs}$ relativamente próximos a zero e $F a 2$ e Cor relativamente próximos a um.

A análise dos resultados mostra uma boa concordância entre os valores calculados no modelo e os dados medidos no experimento. Os melhores resultados foram obtidos utilizando o coeficiente de difusão de Degrazia et al. (2001), para ambos os termos de contragradiente.

Nas Figuras 1, 2 e 3 são apresentados os gráficos de espalhamento entre as concentrações observadas no experimento de Copenhagen em função das concentrações preditas pelo modelo, utilizando os dois termos de contragradiente. As soluções foram avaliadas utilizando as seguintes parametrizações para o coeficiente de difusão vertical: Degrazia et al. (1997) na Figura 1, Degrazia et al. (2001) na Figura 2 e Pleim e Chang (1992) na Figura 3. Podemos notar uma boa conformidade dos dados preditos pelo modelo com os dados observados no experimento de Copenhagen.

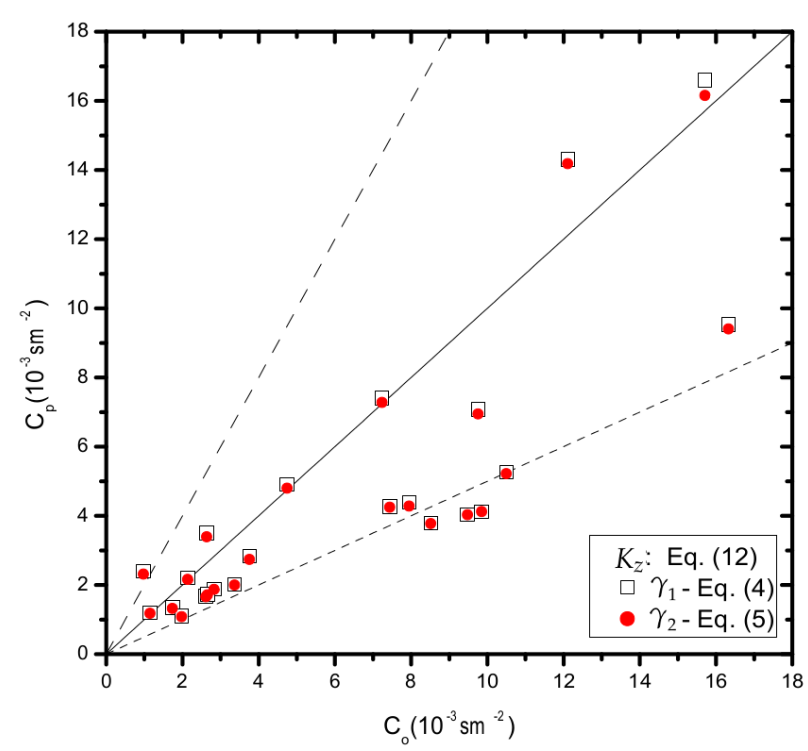

Figura 1: Gráfico de espalhamento entre as concentrações observadas $\left(C_{o}\right)$ e preditas $\left(C_{p}\right)$ pelo modelo para o coeficiente de difusão de Degrazia et al. (1997).

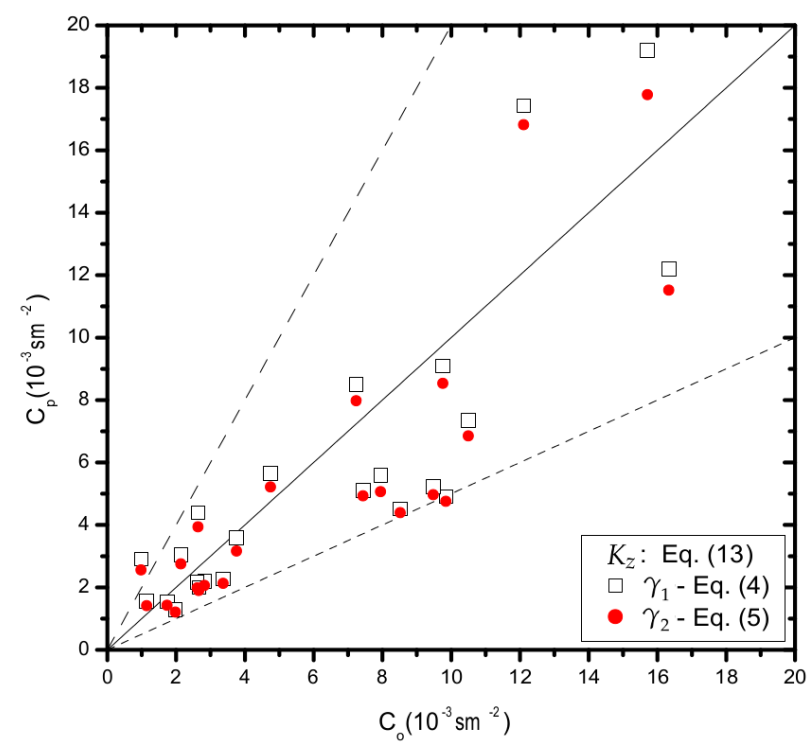

Figura 2: Gráfico de espalhamento entre as concentrações observadas $\left(C_{o}\right)$ e preditas $\left(C_{p}\right)$ pelo modelo para o coeficiente de difusão de Degrazia et al. (2001). 
Tabela 1: Índices Estatísticos do modelo para diferentes coeficientes de difusão

\begin{tabular}{ccccccc}
\hline$K_{z}$ & Contragradiente & Nmse & Cor & Fa2 & Fb & Fs \\
\hline \hline Degrazia et al. & $\gamma_{1}$ - Eq. (4) & 0.30 & 0.837 & 0.826 & 0.300 & 0.131 \\
\cline { 2 - 7 } (1997) - Eq. (12) & $\gamma_{2}$ - Eq. (5) & 0.31 & 0.840 & 0.783 & 0.316 & 0.150 \\
\hline \hline Degrazia et al. & $\gamma_{1}$ - Eq. (4) & 0.20 & 0.856 & 0.913 & 0.156 & -0.003 \\
\cline { 2 - 7 } (2001) - Eq. (13) & $\gamma_{2}$ - Eq. (5) & 0.19 & 0.859 & 0.913 & 0.165 & 0.008 \\
\hline \hline Pleim e Chang & $\gamma_{1}$ - Eq. (4) & 0.31 & 0.815 & 0.783 & 0.281 & 0.077 \\
\cline { 2 - 7 }$(1992)$ - Eq. (14) & $\gamma_{2}$ - Eq. (5) & 0.32 & 0.819 & 0.783 & 0.294 & 0.094 \\
\hline
\end{tabular}

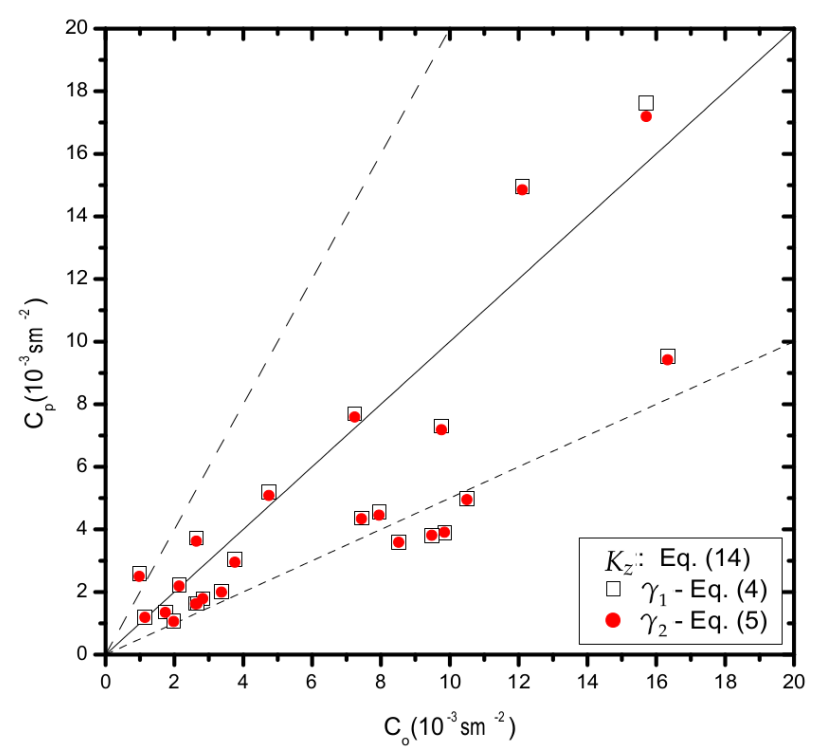

Figura 3: Gráfico de espalhamento entre as concentrações observadas $\left(C_{o}\right)$ e preditas $\left(C_{p}\right)$ pelo modelo para o coeficiente de difusão de Pleim e Chang (1992).

\section{Conclusões}

As duas formulações para o contragradiente, Cuijpers e Holtslag (1998) e Roberti et al. (2004), quando empregadas na equação de advecção-difusão tridimensional em estado estacionário apresentaram um bom desempenho, sem diferenças significativas entre os dois termos na solução e nos resultados obtidos. Os termos caracterizam o transporte não-local da dispersão de poluentes.

O desempenho da solução foi mostrado utilizando algumas parametrizaações da turbulência na CLC e comparado com valores medidos durante o experimento de Copenhagen. A análise dos resultados obtidos e dos índices estatísticos mostram uma boa concordância para os valores da concentração ao nível do solo, entre os valores calculados pelo modelo e os valores experimentais, para ambos os termos de contragradiente.

Dessa forma, no fechamento não-local da turbulência, na equação de advecção-difusão, podemos utlizar para o termo de contragradiente as parametrizações propostas tanto por Cuijpers e Holtslag (1998) quanto por Roberti et al. (2004), pois ambas descrevem de forma satisfatória a dispersão de poluentes na atmosfera.

\section{Agradecimentos}

Os autores agradecem a FAPERGS pelo suporte financeiro neste trabalho.

\section{Referências}

Abate, J., Valkó, P. (2004). Multi-precision laplace transform inversion. Internacional Journal for Numerical Methods in Engineering, 60, 979-993.

Arya, S. (1999). Air Pollution Meteorology and Dispersion. Oxford University Press, New York.

Burden, R. L., Faires, J. D. (2003). Análise Numérica. Thomson, São Paulo.

Costa, C. P., Vilhena, M. T., Moreira, D. M., Tirabassi, T. (2006). Semi-analytical solution of the steady threedimensional advection-diffusion equation in the planetary boundary layer. Atmospheric Environment, 40, 5659-5669.

Cuijpers, J. W. M., Holtslag, A. A. M. (1998). Impact of skewness and nonlocal effects on scalar and buoyancy fluxes in convective boundary layers. Journal of the Atmospheric Sciences, 55, 151-162.

Deardorff, J. W. (1966). The counter-gradient heat flux in the lower atmosphere and in the laboratory. Journal of the Atmospheric Sciences, 23, 503-506.

Deardorff, J. W. (1972). Theoretical expression for the countergradient vertical heat flux. Journal of Geophysical Research, 77, 5900-5904.

Degrazia, G. A., Rizza, U., Mangia, C., Tirabassi, T. (1997). Validation of a new turbulent parameterization for dispersion models in convective conditions. Boundary-Layer Meteorology, 85(2), 243-254. 
Degrazia, G. A., Moreira, D. M., Vilhena, M. T. (2001). Derivation of an eddy diffusivity depending on source distance for vertically inhomogeneous turbulence in a convective boundary layer. Journal of Applied Meteorology, pp. 1233-1240.

Gryning, S. E., Lyck, E. (2002). The Copenhagen Tracer Experiments: Reporting of Measurements. Riso National Laboratory.

Hanna, S. (1989). Confidence limits for air quality models, as estimated by bootstrap and jackknife resampling methods. Atmospheric Environment, 23, 13851395.

Irwin, J. S. (1979). A theoretical variation of the wind profile power-law exponent as a function of surface roughness and stability. Atmospheric Environment, 13(1), 191194.

Panofsky, A. H., Dutton, A. J. (1984). Atmospheric Turbulence. John Wiley \& Sons, New York.

Pleim, J., Chang, J. (1992). A non-local closure model for vertical mixing in the convective boundary layer. Atmospheric Environment, 26A(6), 965-981.

Roberti, D. R., Campos Velho, H. F., Degrazia, G. A. (2004). Identifing counter-gradient term in atmospheric convective boundary layer. Inverse Problems in Science and Engineering, 12(3), 329-339.

Sorbjan, Z. (1989). Structure of the Atmospheric Boundary Layer. Prentice Hall. 